\title{
Theory and Practice
}

\author{
Keith F. Woeltje, MD, PhD
}

In theory there is no difference between theory and practice. But, in practice, there is.

-Jan L. A. van de Snepshcheut, computer scientist and educator (1953-1994)

Surgical site infections (SSIs), with their attendant morbidity and mortality, remain a significant challenge in infection control. To reduce the rates of SSIs, we first need to be able to determine those rates accurately, so that we can determine whether any intervention actually had the desired impact. The theory is easy-simply count the number of procedures done, determine which patients had infections, do a little division, and you're done. But as all healthcare epidemiologists know, the actual practice is not nearly so straightforward. Challenges include determining what constitutes an SSI, sifting through the large numbers of patients involved, and applying appropriate case finding methods. Moving beyond the level of individual hospitals, there are difficult issues in comparing rates between institutions.

Regarding the definition of an SSI, a wide variety of definitions could be defended as reasonable choices. However, as a practical matter, many hospitals in the United States and around the world have settled on the definitions employed by the Centers for Disease Control and Prevention National Nosocomial Infections Surveillance system (NNIS). ${ }^{1}$ Although still subject to some interpretation, these definitions offer a reasonable standard for hospitals to follow.

Dealing with the large number of surgical patients remains an issue for SSI surveillance. Most hospitals handle this, in part, by only performing surveillance for selected surgical procedures. These typically include high-volume procedures, procedures that have had high infection rates at that institution, and procedures for which SSI is particularly problematic if it does develop. Even after a reduction in the number of patients for whom surveillance is performed, case finding represents a significant fraction of an infection control practitioner's (ICP's) time. Institutions are constantly balancing ways of reducing the amount of work involved without sacrificing the quality of surveillance.

The issue of improving the efficiency of case finding is addressed by in this issue of the journal by Chalfine et al. ${ }^{2}$ These investigators developed a computer-assisted surveil- lance system to import microbiologic culture data, surgical procedure data, and general demographic information into a database. Records for patients who had undergone a surgical procedure and had a culture positive for a pathogen were reviewed by an ICP. If the culture specimen came from a surgical wound and the surgical procedure was one for which follow-up was being done, then the surgeon was sent a questionnaire to indicate whether the patient did have an SSI. Before the system was instituted, the surgeons had agreed to participate and were trained in how to answer the questionnaire. To ensure that specimens were appropriately labeled as being from a surgical site, a new ordering form for requesting microbiologic testing was introduced. Chalfine et al. ${ }^{2}$ report data for a 1-year study period on a gastrointestinal and general surgery ward, comparing the SSIs found by the computer-assisted surveillance system and the SSIs found by traditional surveillance, which involved retrospective record review, with each record reviewed by 2 ICPs. The time spent on surveillance was also compared. During the course of the year, 807 procedures that met the criteria for surveillance were performed. Of these 807 procedures, 692 subsequently were associated with cultures positive for a pathogen; 197 (28.5\%) of the cultures were of a specimen from a surgical site. Excluding duplicates, there were 134 potential cases of SSI. Surgeons returned questionnaires for 127 (95\%) of these potential cases, confirming 27 SSIs from the reporting facility. The reference method of surveillance detected 32 SSIs. Of the 5 potential cases of SSI missed, 2 had mislabeled microbiologic culture samples, and 3 had no specimens obtained for culture. The computer-assisted surveillance system had a sensitivity of $84.3 \%$. The time spent on the computer-assisted surveillance system was 90 hours, compared with 223 hours for the reference method, for a reduction in time of $61 \%$.

As Chalfine et al. ${ }^{2}$ acknowledge, the use of computers to screen microbiology data to assist ICPs in finding SSI cases is not new, ${ }^{3,4}$ and neither is sending questionnaires to surgeons to check for SSIs. ${ }^{5}$ Nevertheless, these investigators 
combined the 2 techniques in a fairly unique way to reduce the number of questionnaires sent without sacrificing the sensitivity of surveillance. They demonstrated that there was a significant time savings with their computer-assisted surveillance system; however, their "gold standard" involved review of records for each surgery by 2 ICPs, which inflated the time needed for that approach. On the other hand, as described, their computer-assisted system still appeared to involve a significant amount of ICP intervention. With further automation, the computer-assisted surveillance system might fare very well, compared with chart review by only a single ICP. Chalfine et al. ${ }^{2}$ seemed to have significant cooperation from the surgeons involved on this one unit. Their method also relies on appropriate identification of the source of a microbiologic culture sample in order to work. Thus, it remains to be seen whether their methods can be extended to other facilities. Even if this exact technique cannot be implemented at other institutions, studies such as this add to our understanding of how to make case finding for SSIs more efficient by using available electronic information.

One of the largest challenges in SSI case finding is postdischarge surveillance. The article in this issue by Manniën et al. ${ }^{6}$ describes the impact of postdischarge surveillance on the reported rates of SSI in a large Dutch hospital cohort. The Dutch national nosocomial surveillance network, PREZIES (Preventie van Ziekenhuisinfecties door Surveillance), recommended either placement of a card in a patient's outpatient medical record for completion by the surgeon at the time of outpatient follow-up or review of the outpatient medical record. Hospitals in the network reported whether they used the method recommended by PREZIES, an alternative method of postdischarge surveillance, or no active postdischarge surveillance. Participation in the network was good: 64 of 98 Dutch hospitals participated during at least part of the 9-year period studied. Over the course of the reporting period, the proportion of hospitals predominately using the recommended method increased from $24 \%$ to $50 \%$. Nevertheless, overall, only $24 \%$ of reported surgical procedures had a recommended method of postdischarge surveillance performed, $25 \%$ had an alternative active postdischarge surveillance performed, and $52 \%$ had only passive postdischarge surveillance performed. Not surprisingly, when recommended postdischarge surveillance was used, the percentage of SSIs that were discovered after discharge and the overall SSI rate increased.

Unfortunately, the PREZIES system did not collect data on which of the 2 recommended methods was used, so it is unclear how successful the practice of having surgeons fill out individual cards was. Clearly, without some form of postdischarge surveillance, patients with SSI will be missed, and SSI rates will be underestimated. Review of patient medical records is extremely labor-intensive, ${ }^{7}$ and reliance on less time-consuming methods of postdischarge surveillance (eg, questionnaires mailed to physicians) may not be very sensitive. ${ }^{8}$ Insurance claims data may provide an alternative method for postdischarge SSI surveillance, ${ }^{9}$ but this information may not be readily available to most hospitals. The issue of how best to perform postdischarge SSI surveillance remains unresolved.

A third article on SSIs in this issue of the journal focuses not on case finding but on another issue of practical importance: patient risk stratification. Batista et al..$^{10}$ describe incorporating a medication-based measure of patient comorbidity in the assessment of risk for development of an SSI following coronary artery bypass graft (CABG) surgery. The current NNIS risk adjustment system uses the American Society of Anesthesiologists (ASA) score as its only patient comorbidity component. NNIS adds 1 point to its score for ASA scores of 3 or greater; because most patients who require CABG fall into this category, they are not well differentiated for risk adjustment purposes. Batista et al. ${ }^{10}$ calculated an admission chronic disease score (ACDS) for patients undergoing CABG. The ACDS infers comorbidities from the medications prescribed to a patient on the day of admission to the hospital. ${ }^{11,12}$ Because patients who had surgery on the day of admission received standard medications at the study institution, a variant of the ACDS, termed the preadmission ACDS (PACDS) was calculated on the basis of the patient's preadmission medications as listed on the discharge summary. In a case-control study, the NNIS risk index and the ASA score alone were not significant predictors of SSI. The ACDS and PACDS were used as components of a modified NNIS index (assigning 1 point if the score was greater than a stated threshold). When this was done, the NNIS-ACDS and NNIS-PACDS scores were more highly correlated with the risk of SSI than was the standard NNIS-ASA score; however, the correlations were not statistically significant. In logistic regression modeling, the ACDS, the PACDS, the highest PACDS quintile, and a NNIS-PACDS score of 2 were all significant predictors of post-CABG SSI.

The study by Batista et al. ${ }^{10}$ extends previous work by this study group. ${ }^{12}$ It is clear that for many types of surgery, better risk adjustment models are needed. The ACDS/PACDS approach needs to be validated with other types of surgery and at other institutions, but it may provide a more useful measure of comorbidities than does the ASA score. As Batista et al. ${ }^{10}$ point out, one of the biggest difficulties with this approach is determining what medications the patient received. This information is most easily obtained if hospitals have access to electronic pharmacy data. As more hospitals implement computerized physician-order entry systems, this information may become more readily available. In addition, the Joint Commission on Accreditation of Healthcare Organizations patient-safety goal of achieving better medication reconciliation ${ }^{13}$ means that more attention is being given to ensuring that an accurate record of a patient's preadmission medications is being obtained by hospitals.

Taken together, these 3 articles, and the other articles on the subject of SSI in this issue of the journal, contribute to our understanding of how to put the theory of SSI surveil- 
lance into practice. Although many hospitals use the same definitions for SSIs, clearly methods of case finding and postdischarge surveillance vary tremendously between institutions. And once an optimal method for case finding has been identified, optimal methods for risk adjustment still need to be defined. Until recently, such issues have been somewhat academic. What was important was that each institution remain consistent in how cases were found and how definitions were applied. This allowed the institution to monitor its own SSI rates over time. With the increasing availability of electronic data on patients, novel surveillance methods have begun to be developed ${ }^{14,15}$ that can monitor SSI rates without a significant investment of ICP time; these methods may not give the true rate, but as long as they were consistent over time, they could serve, for surveillance purposes. By reducing the burden of surveillance, such novel methods might provide ICPs with more time for interventions to prevent SSIs.

The recent increase in the United States of legislation that mandates reporting of healthcare-associated infections makes the variability in definitions, case finding, postdischarge surveillance, and risk adjustment much more problematic. ${ }^{16}$ Some states, such as Missouri, have required the use of NNIS definitions. However, case finding methods (including postdischarge surveillance) continue to vary between institutions. Methods that work well at one hospital may not work at all at another (eg, because of differences in hospital culture, information systems, the ease of access to outpatient records), so it is likely that it will never be possible to establish a standard surveillance method. Even if SSIs could be found consistently, the ability to perform risk adjustment for patient populations at different hospitals remains suboptimal. Thus, comparison of SSI rates between institutions is fraught with problems. In theory, mandatory reporting of infections is good, because it will provide hospitals with incentives to improve their processes. In practice, mandatory reporting may provide disincentives for hospitals to explore new ways of improving surveillance for SSIs. Having states mandate particular methods might further inhibit innovation in this area. Being forced to compare rates, however, will encourage research into better risk-adjustment models.

In theory, healthcare epidemiologists will continue to strive for better methods of SSI surveillance. I'm optimistic that this will continue in practice as well.

Address reprint requests to Keith F. Woeltje, $\mathrm{MD}, \mathrm{PhD}$, Division of Infectious Diseases, Washington University School of Medicine, Campus Box 8051, 660 S. Euclid Ave., St. Louis, MO 63110-1094 (kwoeltje@im.wustl.edu).

\section{REFERENCES}

1. Horan TC, Gaynes RP. Surveillance of nosocomial infections, Appendix A: CDC definitions of nosocomial infections (excluding pneumonia). In: Mayhall CG, ed. Hospital Epidemiology and Infection Control. 3rd ed. Philadelphia; Lippincott Williams \& Wilkins; 2004:1672-1685.

2. Chalfine A, Cauet D, Lin WC, et al. Highly sensitive and efficient computer-assisted system for routine surveillance of surgical site infections. Infect Control Hosp Epidemiol 2006; 27:794-801 (in this issue).

3 Pokorny L, Rovira A, Martin-Baranera M, Gimeno C, Alonso-Tarres C, Vilarasau J. Automatic detection of patients with nosocomial infection by a computer-based surveillance system: a validation study in a general hospital. Infect Control Hosp Epidemiol 2006; 27:500-503.

4 Evans RS, Larsen RA, Burke JP, et al. Computer surveillance of hospitalacquired infections and antibiotic use. JAMA 1986; 256:1007-1011.

5 Manian FA, Meyer L. Adjunctive use of monthly physician questionnaires for surveillance of surgical site infections after hospital discharge and in ambulatory surgical patients: report of a seven-year experience. Am J Infect Control 1997; 25:390-394.

6. Manniën J, Wille JC, Snoeren RL, van den Hof S. The impact of postdischarge surveillance on surgical site infection rates for several surgical procedures; results from the nosocomial surveillance network in The Netherlands. Infect Control Hosp Epidemiol 2006; 27:809-816 (in this issue).

7. Hueffmeier A, Payton M, McMullen K, Woeltje KF. Developing an outpatient surgical site infection surveillance system at a tertiary care medical center. In: Program and abstracts of the 16th Annual Scientific Meeting of the Society for Healthcare Epidemiology of America; March 2006; Chicago, IL. Abstract 164.

8. Sands K, Vineyard G, Platt R. Surgical site infections occurring after hospital discharge. J Infect Dis 1996; 173:963-970.

9. Sands K, Vineyard G, Livingston J, Christiansen C, Platt R. Efficient identification of postdischarge surgical site infections: use of automated pharmacy dispensing information, administrative data, and medical record information. J Infect Dis 1999; 179:434-441.

10. Batista R, Kaye K, Yokoe DS. Admission-based chronic disease scores as alternative predictors of surgical site infection for patients undergoing coronary artery bypass graft surgery. Infect Control Hosp Epidemiol 2006; 27:802-808 (in this issue).

11. Clark DO, Von Korff M, Saunders K, Baluch WM, Simon GE. A chronic disease score with empirically derived weights. Med Care 1995; 33:783-95.

12. Kaye KS, Sands K, Donahue JG, et al. Preoperative drug dispensing as predictor of surgical site infection. Emerg Infect Dis 2001; 7:57-65.

13 Joint Commission on Accreditation of Healthcare Organizations, USA. National Patient Safety Goals for 2006. Available at: http://www .jointcommission.org/Standards/NationalPatientSafetyGoals/. Accessed June 8, 2006.

14. Leth RA, Moller JK. Surveillance of hospital-acquired infections based on electronic hospital registries. J Hosp Infect 2006; 62:71-79.

15. Sands K, Vineyard G, Livingston J, Christiansen C, Platt R. Efficient identification of postdischarge surgical site infections: use of automated pharmacy dispensing information, administrative data, and medical record information. J Infect Dis 1999; 179:434-441.

16. Wong ES, Rubb ME, Mermel L, et al. Public disclosure of healthcareassociated infections: the role of the Society for Healthcare Epidemiology of America. Infect Control Hosp Epidemiol 2005; 26:210-212. 